\title{
Changes in short- and long-term cardiovascular risk of incident diabetes and incident myocardial infarction-a nationwide study
}

\author{
M. L. Norgaard • S. S. Andersen • T. K. Schramm • F. Folke • C. H. Jørgensen • \\ M. L. Hansen • C. Andersson • D. M. Bretler • A. Vaag • L. Kober • C. Torp-Pedersen • \\ G. H. Gislason
}

Received: 11 February 2010 /Accepted: 13 April 2010 /Published online: 9 May 2010

(C) Springer-Verlag 2010

\begin{abstract}
Aims/hypothesis We assessed secular trends of cardiovascular outcomes following first diagnosis of myocardial infarction (MI) or diabetes in an unselected population.

Methods All Danish residents aged $\geq 30$ years without prior diabetes or MI were identified by individual-level linkage of nationwide registers. Individuals hospitalised with MI or claiming a first-time prescription for a glucose-lowering medication (GLM) during the period from 1997 to 2006 were included. Analyses were by Poisson regression models. Primary endpoints were death by all causes, cardiovascular death and MI.

Results The study included 3,092,580 individuals, of whom 77,147 had incident MI and 118,247 new-onset diabetes. MI patients had an increased short-term risk of all endpoints compared with the general population. The rate ratio (RR) for cardiovascular death within the first year
\end{abstract}

M. L. Norgaard $(\bowtie) \cdot$ S. S. Andersen $\cdot$ F. Folke

C. H. Jørgensen • M. L. Hansen • C. Andersson • D. M. Bretler •

C. Torp-Pedersen • G. H. Gislason

Department of Cardiology, Gentofte Hospital,

Niels Andersens Vej 65,

DK 2900 Hellerup, Denmark

e-mail: mln@heart.dk

T. K. Schramm $\cdot$ L. Køber

The Heart Centre, Rigshospitalet, University of Copenhagen,

Copenhagen, Denmark

L. Køber $\cdot$ C. Torp-Pedersen

Faculty of Health Sciences, University of Copenhagen,

Copenhagen, Denmark

A. Vaag

Steno Diabetes Center,

Gentofte,

Copenhagen, Denmark after MI was 11.1 (95\% CI 10.8-11.5) in men and 14.8 (14.3-15.3) in women, respectively. The risk rapidly declined and 1 year after the index MI, RR was 2.11 (2.00-2.23) and 2.8 (2.64-2.97) in men and women, respectively. Patients with diabetes carried a constantly elevated risk of all endpoints compared with the general population. The cardiovascular death RR was 1.90 (1.77$2.04)$ and 1.92 (1.78-2.07) in men and women, respectively during the first year after GLM initiation.

Conclusions/interpretation Incident MI is associated with high short-term risk, which decreases rapidly over time. Incident diabetes is associated with a persistent excessive cardiovascular risk after initiation of GLM therapy. This further strengthens the necessity of early multi-factorial intervention in diabetes patients for long-term benefit.

Keywords Cardiovascular risk · Coronary risk · Diabetes mellitus · Epidemiology · Long-term risk · Mortality ·

Prognosis $\cdot$ Short-term risk

$\begin{array}{ll}\text { Abbreviations } \\ \text { GLM } & \text { Glucose-lowering medication. } \\ \text { ICD } & \text { International Classification of Diseases } \\ \text { MI } & \text { Myocardial infarction } \\ \text { RR } & \text { Rate ratio } \\ \text { UKPDS } & \text { UK Prospective Diabetes Study }\end{array}$

\section{Introduction}

Patients with diabetes requiring glucose-lowering treatment have been reported as having the same long-term risk of major cardiovascular outcomes as patients with a previous myocardial infarction (MI) [1,2]. This increase in risk in 
diabetes patients is independent of age and sex, and applies to all patients with type 1 as well as those with type 2 diabetes [1].

Population studies have shown that: (1) the mortality rate from coronary heart disease is doubled in patients with diabetes compared with individuals without diabetes; and (2) that diabetes confers an equivalent risk to that conferred by 15 years of ageing when estimating cardiovascular risk $[3,4]$. A decline in all-cause and cardiovascular mortality rates has been observed over the last decades among diabetes patients and individuals without diabetes. However, the decline in mortality rates in diabetes patients appears to be markedly lower than in individuals without diabetes $[5,6]$. Thus, diabetes patients remain at high risk of allcause and cardiovascular mortality [5].

The similarity in cardiovascular risk for patients with diabetes and for patients with a previous $\mathrm{MI}$ is one reason why guidelines recommend similar preventive strategies to reduce risk [7]. To date, studies comparing risk in patients with MI or diabetes have not focused on the time that has elapsed since diagnosis. The risk would be expected to change over time for patients with MI as well as for those with diabetes. To further assess the risk in the two patient groups, it is necessary to compare the change in risk over time following the diagnosis.

To establish a more detailed comparison of major cardiovascular events following a diagnosis of diabetes or MI, we conducted a nationwide study examining short- and long-term mortality rates in patients with incident diabetes and patients with first-time MI during a 10 year period in Denmark, using the background population as a reference.

\section{Methods}

Population and data sources All residents in Denmark have a unique and permanent civil registration number that enables individual-level linkage between nationwide administrative registers. Using the Danish Civil Registration system, we identified all inhabitants in Denmark who were at least 30 years of age and alive on 1 January 1997. All subsequent medications dispensed from pharmacies were obtained from the National Prescription Register (the Danish Register of Medicinal Product Statistics), which includes all prescriptions dispensed from Danish pharmacies since 1995. The prescription register keeps information on date of dispensing, strength and quantity. Information on hospital admissions was obtained from the Danish National Patient Register [8], which keeps records on all hospital admissions in Denmark since 1978. Each hospitalisation is registered at discharge with diagnoses recorded using the International Classification of Diseases (ICD). Until 1994 the 8th revision (ICD-8) was used and from 1994 the 10th revision (ICD-10; www.who.int/classifications/icd/en/, accessed 5 January 2009). All deaths were identified from the Central Population Register, where fatalities are recorded within 2 weeks. Causes of death were obtained from the National Causes of Death Register, which records immediate and contributing causes using the ICD- 8 and ICD-10.

The reference population comprised inhabitants in Denmark of at least 30 years of age and alive on 1 January 1997. Patients with a prior history of MI or patients receiving prescriptions for glucose-lowering medication (GLM) before 1 January 1997 were excluded from the study.

Myocardial infarction and diabetes mellitus Incident MI was identified as first-time hospitalisation with MI. The diagnosis of MI in the National Patient Register has proven to be valid, with a sensitivity of $91 \%$ and a positive predictive value of 93\% [9]. Patients with incident diabetes requiring glucose-lowering treatment were identified as individuals initiating GLM (oral or insulin) during the study period (1 January 1997 to 31 December 2006) [1]. Patients were registered as either incident $\mathrm{MI}$ or incident diabetes depending on which diagnosis came first.

Comorbidity Comorbidity was assessed by registration of pre-specified hospital discharge diagnoses up to 1 year before the inclusion date (date of the incident), as listed in Table 1. For the reference population comorbidity was assessed 1 year prior to 30 June 2001 for individuals surviving beyond that date and 1 year prior to 1 January 1997 for individuals who died before 30 June 2001, respectively.

Outcomes The predefined endpoints of interest were: allcause mortality, cardiovascular death (ICD-10 codes I00-I99) and new onset MI (ICD-10 codes I21, I22).

Statistical analyses Continuous variables are presented as mean with standard deviation (SD). Categorical data are presented as percentages.

Univariate presentations of endpoints were performed using Kaplan-Meier estimates of event risk vs time during selected time periods. Multivariable analysis of risk was performed using Poisson regression analysis, adjusted for age, sex and calendar year.

Multivariable analyses revealed no interaction between age and the different risk groups, but there was significant interaction between sex and calendar time. Consequently the study period was divided into two time periods: 1 January 1997 to 30 June 2001 and 1 July 2001 to 31 December 2006, hereafter denoted as early and late period, respectively. The two study periods and the two sexes were analysed separately. 
All statistical calculations were performed with SAS statistical software package, version 9.1 (SAS Institute, Cary, NC, USA).

Ethics The Danish Data Protection Agency approved the study (no. 2007-41-1667). Retrospective register studies do not require ethical approval in Denmark.

\section{Results}

On 1 January 1997, 3,092,580 individuals aged 30 years or more and without prior diabetes or MI resided in Denmark. Of these, 77,147 (2.5\%) individuals were hospitalised with incident MI during the study period and 118,247 (3.8\%) initiated treatment with GLM. Demographic characteristics are summarised in Table 1.

All-cause mortality There was a high short-term risk of death in the incident MI population in the early period and late period for both sexes compared with the reference population (Table 2). The risk rapidly declined, remaining stable from 1 year after MI for the rest of the study period. The risk was consistently higher in women than in their male counterparts.
The initial risk of death in the incident diabetes population was increased by more than twofold compared with the reference population. In women with incident diabetes, the risk remained stable at approximately 1.5 -fold higher than in the reference population. In men, there was a trend towards risk reduction 1 year after onset of diabetes.

Cardiovascular death The incident MI population initially carried a more than tenfold excessive risk of cardiovascular mortality compared with the reference population (Table 2). The risk rapidly decreased, reaching an approximately threefold higher risk in women and a twofold higher risk in men in both periods from 1 year after the incident MI, compared with the reference population (Table 2, Fig. 1a, b). There was no trend towards a risk reduction in either sex when comparing the two study periods.

The incident diabetes population had an initial twofold higher risk of cardiovascular death in both periods applying for both sexes compared with the reference population. For women, the risk was nearly constant and no trend towards risk reduction was demonstrated when comparing the two study periods. In men with incident diabetes, a trend towards risk reduction was demonstrated for the late period compared with the early period from 1 year after the incident (Table 2, Fig. 1a, b).

Table 1 Demographic characteristics according to exposure-group and time period

\begin{tabular}{|c|c|c|c|c|c|}
\hline \multirow[t]{2}{*}{ Variable } & \multicolumn{2}{|l|}{ Incident MI } & \multicolumn{2}{|c|}{ Incident diabetes } & \multirow[t]{2}{*}{ Reference } \\
\hline & Early period $^{\mathrm{a}}$ & Late period ${ }^{\mathrm{b}}$ & Early period $^{\mathrm{a}}$ & Late period ${ }^{\mathrm{b}}$ & \\
\hline \multicolumn{6}{|l|}{ Participants } \\
\hline All $(n)$ & 33,378 & 43,769 & 49,199 & 69,048 & $2,897,186$ \\
\hline $\operatorname{Men}(n)$ & 20,463 & 26,560 & 26,992 & 38,223 & $1,364,120$ \\
\hline Women $(n)$ & 12,915 & 17,209 & 22,207 & 30,825 & $1,533,066$ \\
\hline Male sex $(\%)$ & 61 & 61 & 55 & 55 & 54 \\
\hline \multicolumn{6}{|l|}{ Age (years) ${ }^{\mathrm{c}}$} \\
\hline All participants & $69.7(13.0)$ & $70.5(13.3)$ & $62.7(13.6)$ & $63.0(12.5)$ & $52.7(15.7)$ \\
\hline Men & $66.9(12.8)$ & $67.5(12.9)$ & $61.0(12.9)$ & $61.7(11.8)$ & $51.1(14.7)$ \\
\hline Women & $74.2(12.1)$ & $75.1(12.5)$ & $64.8(14.1)$ & $64.7(13.1)$ & $54.1(16.4)$ \\
\hline \multicolumn{6}{|l|}{ Prior hospital admissions ${ }^{\mathrm{d}}$} \\
\hline COPD (\%) & 5.8 & 7.4 & 2.3 & 2.4 & 0.3 \\
\hline Renal disease (\%) & 1.5 & 2.5 & 0.4 & 0.4 & 0.05 \\
\hline Cancer $(\%)$ & 2.9 & 3.4 & 2.5 & 2.9 & 0.5 \\
\hline Congestive heart failure $(\%)$ & 11.9 & 12.3 & 2.2 & 2.0 & 0.2 \\
\hline
\end{tabular}

Values are mean $(\mathrm{SD})$ unless otherwise indicated

${ }^{a}$ Time period 1 January 1997 to 30 June 2001

${ }^{\mathrm{b}}$ Time period 1 July 2001 to 31 December 2006

${ }^{\mathrm{c}}$ Defined on 1 January 1997

${ }^{\mathrm{d}}$ Hospitalisation up to 1 year before incident event; for the reference population the date was set to 1 year prior to 30 June 2001, unless death occurred before that date, in which case date was set to 1 year prior to 1 January 1997

COPD, chronic obstructive pulmonary disease 
Table 2 Age-adjusted Poisson regression analyses using the background population as a reference
Values are RR $(95 \% \mathrm{CI})$ unless otherwise indicated

${ }^{\text {a }}$ Time period 1 January 1997 to 30 June $2001 ;{ }^{b}$ time period 1 July 2001 to 31 December 2006

\begin{tabular}{|c|c|c|c|c|}
\hline \multirow[t]{2}{*}{ Variable } & \multicolumn{2}{|l|}{ Incident MI } & \multicolumn{2}{|l|}{ Incident diabetes } \\
\hline & Early period $^{\mathrm{a}}$ & Late period ${ }^{\mathrm{b}}$ & Early period $^{\mathrm{a}}$ & Late period ${ }^{\mathrm{b}}$ \\
\hline \multicolumn{5}{|c|}{ All-cause mortality } \\
\hline Events $(n)$ & 17,360 & 14,230 & 15,341 & 7,704 \\
\hline \multicolumn{5}{|c|}{ RR $0-1$ years } \\
\hline Men & $6.46(6.27-6.65)$ & $6.17(5.98-6.36)$ & $2.23(2.13-2.33)$ & $2.29(2.18-2.93)$ \\
\hline Women & $8.53(8.27-8.79)$ & $8.67(8.41-8.94)$ & $2.16(2.05-2.27)$ & $2.51(2.40-2.64)$ \\
\hline \multicolumn{5}{|c|}{ RR 1-3 years } \\
\hline Men & $1.42(1.36-1.49)$ & $1.47(1.39-1.55)$ & $1.63(1.57-1.70)$ & $1.42(1.35-1.50)$ \\
\hline Women & $1.90(1.81-2.00)$ & $2.02(1.91-2.15)$ & $1.50(1.43-1.57)$ & $1.49(1.41-1.58)$ \\
\hline \multicolumn{5}{|c|}{ RR 3-5 years } \\
\hline Men & $1.38(1.31-1.45)$ & $1.46(1.32-1.62)$ & $1.59(1.52-1.66)$ & $1.42(1.29-1.56)$ \\
\hline Women & $1.84(1.74-1.94)$ & $1.80(1.60-2.02)$ & $1.51(1.44-1.58)$ & $1.42(1.27-1.58)$ \\
\hline \multicolumn{5}{|c|}{ Cardiovascular death } \\
\hline Events $(n)$ & 14,508 & 11,923 & 8,486 & 3,636 \\
\hline \multicolumn{5}{|c|}{ RR $0-1$ years } \\
\hline Men & $11.1(10.8-11.5)$ & $10.4(10.1-10.8)$ & $1.90(1.77-2.04)$ & $1.97(1.84-2.12)$ \\
\hline Women & $14.8(14.3-15.3)$ & $14.8(14.3-15.3)$ & $1.92(1.78-2.07)$ & $2.10(1.94-2.28)$ \\
\hline \multicolumn{5}{|c|}{ RR 1-3 years } \\
\hline Men & $2.11(2.00-2.23)$ & $2.14(2.00-2.28)$ & $1.79(1.69-1.88)$ & $1.51(1.40-1.63)$ \\
\hline Women & $2.80(2.64-2.97)$ & $2.92(2.72-3.13)$ & $1.70(1.60-1.80)$ & $1.67(1.54-1.81)$ \\
\hline \multicolumn{5}{|c|}{ RR 3-5 years } \\
\hline Men & $1.99(1.88-2.11)$ & $2.10(1.86-2.34)$ & $1.89(1.79-2.00)$ & $1.61(1.41-1.84)$ \\
\hline Women & $2.63(2.46-2.81)$ & $2.77(2.42-3.17)$ & $1.78(1.68-1.89)$ & $1.65(1.42-1.91)$ \\
\hline \multicolumn{5}{|c|}{ MI/recurrent MI } \\
\hline Events $(n)$ & 11,422 & 9,942 & 3,668 & 1,613 \\
\hline \multicolumn{5}{|c|}{ RR 0-1 years } \\
\hline Men & $21.7(21.1-22.4)$ & $20.2(19.5-20.9)$ & $1.78(1.62-1.96)$ & $1.72(1.55-1.90)$ \\
\hline Women & $43.3(41.8-44.9)$ & $42.6(41.1-44.2)$ & $1.93(1.70-2.20)$ & $2.05(1.80-2.34)$ \\
\hline \multicolumn{5}{|c|}{ RR 1-3 years } \\
\hline Men & $2.99(2.80-3.18)$ & $2.92(2.69-3.17)$ & $1.62(1.51-1.74)$ & $1.29(1.16-1.44)$ \\
\hline Women & $5.67(5.25-6.11)$ & $5.64(5.13-6.21)$ & $1.93(1.75-2.12)$ & $1.72(1.51-1.96)$ \\
\hline \multicolumn{5}{|c|}{ RR 3-5 years } \\
\hline Men & $2.67(2.48-2.87)$ & $2.70(2.30-3.17)$ & $1.58(1.46-1.71)$ & $1.33(1.10-1.62)$ \\
\hline Women & $4.33(3.93-4.78)$ & $5.15(4.24-6.25)$ & $1.97(1.78-2.17)$ & $1.89(1.49-2.40)$ \\
\hline
\end{tabular}

Cardiovascular death promptly increased in the incident MI population after the index hospitalisation, but thereafter levelled off with an approximately constant elevated risk. The diabetes group showed a more gradual and progressive increase in risk of cardiovascular death over time; this was comparable to the reference population, although the risk in the diabetes patients was higher at all time points (Fig. 2).

Table 3 demonstrates an increasing rate of MI and diabetes with increasing age in all exposure groups. No significant difference in rate of MI and diabetes was observed in any of the exposure groups during the two study periods.
Myocardial infarction/recurrent myocardial infarction Both men and women with incident MI had a markedly elevated short-term risk of recurrent MI, but the risk reached a steady level after 1 year. There was no trend towards a risk reduction between the two study periods. Women with incident MI carried a higher risk of recurrent MI than men at all time-points (Table 2).

Women with incident diabetes had an approximately twofold higher risk of MI than the reference population, with only a minimal decline in risk over time. Men with incident diabetes had an increased risk of MI (rate ratio [RR] approximately 1.7), which declined moderately over time. A trend towards a risk reduction in men was also 


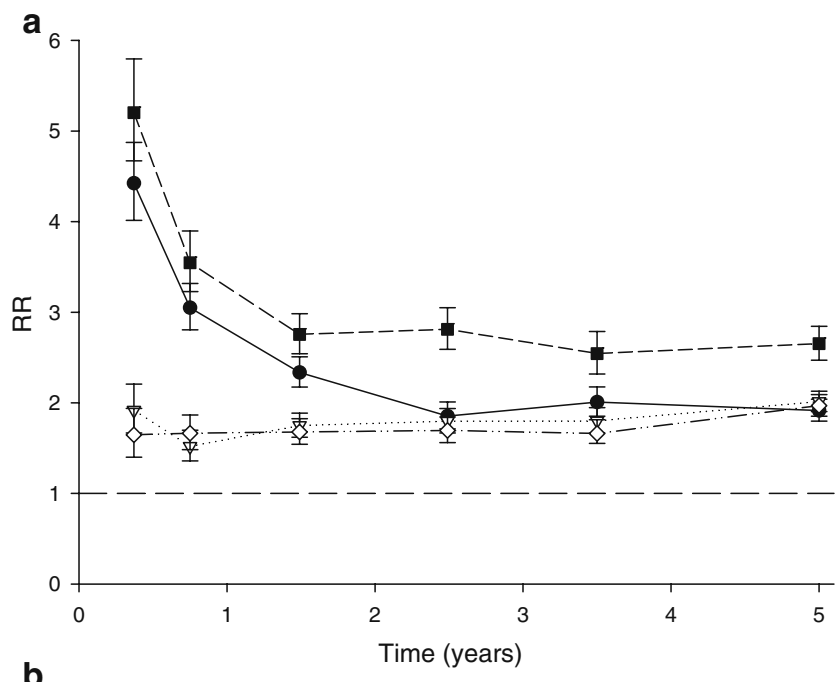

b

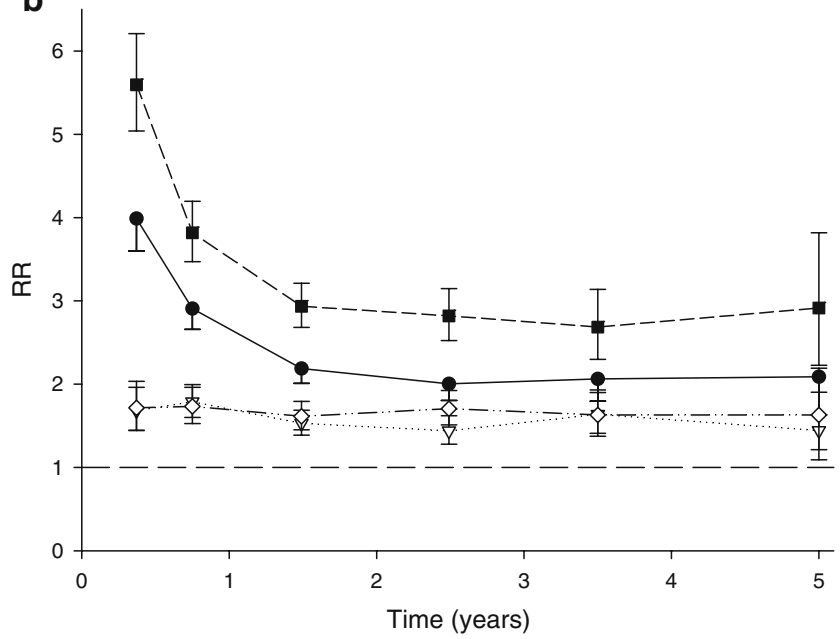

Fig. 1 a Poisson analysis of cardiovascular death according to exposure groups in the early time period stratified by sex. b Poisson analysis as above for late time period stratified by sex. Black circles, incident MI men; black squares, incident MI women; white triangles, incident diabetes men; white diamonds, incident diabetes women; broken line, reference

demonstrated when comparing the two study periods (Table 2).

Sensitivity analysis After exclusion of patients with renal failure and congestive heart failure, we found a modest risk reduction within the 1 st year on all-cause mortality and cardiovascular mortality applying for both sexes in the MI population compared with the background population. Thus all-cause mortality RR in men was 5.81 (95\% CI $5.62-$ $6.00)$ as compared with $6.46(6.27-6.65)$ and 5.24 (5.06$5.43)$ as compared with $6.17(5.98-6.36)$ in the early and late periods, respectively. In women, RR was 7.89 (7.62$8.18)$ as compared with $8.53(8.27-8.79)$ and 7.84 (7.56$8.12)$ as compared with $8.67(8.41-8.94)$ in the early and late period, respectively. No risk reduction was found after the 1 st year after the incident. Similarly, the risk of

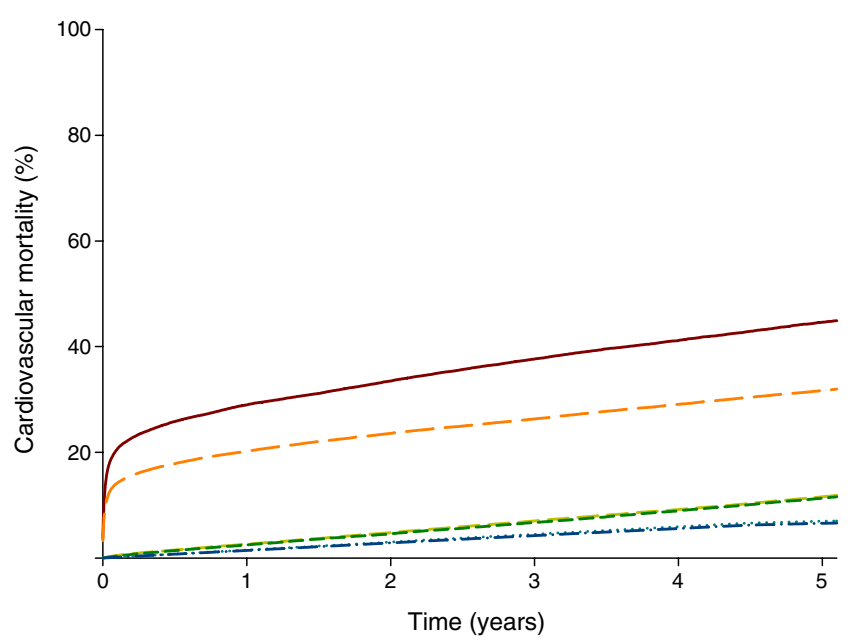

Fig. 2 Unadjusted cardiovascular mortality stratified by sex. Brown line, incident MI women; orange dashed line, incident MI men; pale green dashed line, incident diabetes women; green dashed line, incident diabetes men; light blue dotted line, reference women; blue dashed and dotted line, reference men

cardiovascular mortality in men was RR 10.04 (9.6910.40) as compared with RR $11.1(10.8-11.5)$ and 8.90 (8.56-9.26) as compared with $10.4(10.1-10.8)$ in the early and in the late period, respectively. In women, the RR for cardiovascular mortality was $13.7(13.20-14.22)$ as compared with $14.8(14.3-15.3)$ and $13.39(12.88-13.92)$ as compared with $14.8(14.3-15.3)$ in the early and the late period, respectively. No risk reductions were found in analyses of long-term outcomes (data not shown).

For both endpoints, no risk reduction was documented after excluding renal failure and congestive heart failure patients in the diabetes population.

\section{Discussion}

This nationwide study assessed changes in risk of adverse cardiovascular outcomes in patients with incident diabetes or incident MI, respectively, compared with the reference population. The main finding was an approximately twofold elevated risk of all endpoints immediately following initiation of GLM treatment. The excessive risk persisted after the first year. Men with incident diabetes exhibited a trend towards risk reduction in the late time period compared with the early. There was a high shortterm risk of all endpoints in the incident MI population, with a rapid decline in risk during the 1st year.

Significant improvements in the treatment of acute MI have been implemented during the last decade. In 2003, percutaneous coronary intervention was applied as the gold standard for acute ST-elevation MI in Denmark [10] and the use of beta-blockers, statins and platelet inhibitors has markedly increased [11-14]. However, no decline of 
Table 3 Rates of myocardial infarction and diabetes per 1,000 person-years stratified by age according to exposure group

\begin{tabular}{lccccc}
\hline Variable & \multicolumn{2}{l}{ Incident MI } & & \multicolumn{2}{l}{ Incident diabetes } \\
\cline { 2 - 3 } \cline { 5 - 6 } & $1997-2001^{\mathrm{a}}$ & $2001-2006^{\mathrm{b}}$ & & $1997-2001^{\mathrm{a}}$ & $2001-2006^{\mathrm{b}}$ \\
\hline Age & & & & 2.21 \\
$<55$ years & 0.80 & 1.01 & & 5.85 & 2.94 \\
$56-70$ years & 3.76 & 3.30 & 6.78 & 6.50 \\
$>70$ years & 8.10 & 8.90 & & 6.84 \\
\hline
\end{tabular}

relative risk was demonstrated when comparing the incident MI patients from the two time periods. A possible explanation could be insufficient treatment with betablockers and statins following hospitalisation after MI, especially if treatment is not initiated in close proximity to discharge $[15,16]$. Subsequently, the lack of any risk reduction during the two study periods could partially be explained by the need for a longer lag or observation time after the improved use of secondary prophylactic treatment before the prognosis changes. The results of our study emphasise the need to maintain focus on intensifying the secondary treatment in extension to hospitalisation and post discharge for a first-time MI.

Our study indicates that women have a higher risk of MI than men at all times, even after adjusting for age. Since women tend to have infarctions at an older age than men as well as higher rates of comorbidities, the poorer outcomes in women than in men have largely been attributed to these factors; sex has generally not been interpreted as constituting an independent association [17]. Furthermore, Dominguez et al. have shown that women with diabetes are not treated less frequently with statins than their male counterparts [18]. Since the above-mentioned study did not reveal a convincing sex-related difference in statin treatment, the explanation of the higher risk for MI in women following initiation of GLM remains to be explored. This aspect needs further investigation, taking into account concomitant treatment and possible discrepancies in invasive treatment.

During the two study periods several novel treatment modalities were implemented and new glucose-lowering agents introduced. Specifically, the UK Prospective Diabetes Study (UKPDS) provided convincing evidence that intensive glycaemic control prevents development of microvascular complications in type 2 diabetes patients [19]. In the decade since that study, the use of all GLM, including insulin, alone or in supplement to oral GLM therapy, has increased significantly. Although the initial results from the UKPDS did not conclusively document any beneficial effects of intensified glycaemic control on prevention of macrovascular complications, epidemiological data from the UKPDS supported the possibility that such benefits occur; moreover, recent data have shown a beneficial legacy effect of early intensive glycaemic control on prevention of cardiovascular disease in patients with type 2 diabetes [20]. The Steno 2 study showed that intensive multi-factorial treatment including blood pressure-, glucose- and lipid-lowering therapies, along with lifestyle interventions prevents (or postpones) the development microand macrovascular complications in patients with type 2 diabetes [21, 22]. Together, these findings have been implemented into national and international guidelines over the last decade. However, our finding that there was only a trend towards risk reduction in men, but not in women from the early to the late period may seem surprising and somewhat disappointing. It is possible that the explanation for a risk reduction is related to changes in the treatment of patients with diabetes implemented during the 10 year study period. However, another potential explanation is that, after implementation of the novel guidelines around the millennium, more diabetes patients initiated treatment with GLM earlier, resulting in a lower risk patient population in the later as compared with earlier observation period. If such a change in overall risk in the otherwise well defined segment of the diabetes population requiring GLM has actually occurred, it seems even more surprising that there has not been a greater reduction of mortality rates, questioning the true clinical impact of intensified treatment in patients with diabetes on a population basis. Explanations for this may include a delay in implementation of guidelines, as well as the need for a longer lag or observation time before the prognosis changes. Another explanation may relate to the question of whether the clinical outcome benefits associated with improvement of metabolic control from acceptable to optimal glycaemic levels are marginal or potentially non-existent, as illustrated by the negative outcomes in recent interventional studies [23-25].

Our findings are consistent with results from the Framingham Heart Study [5] demonstrating no reduction of mortality rates in diabetes patients over the last 50 years and a persistent twofold higher mortality rate.

Haffner et al. have demonstrated that patients with diabetes without prior MI exhibit the same risk of MI as patients without diabetes, but with prior MI [2]. Schramm et al. confirmed those results in a nationwide cohort of diabetes patients and provided evidence that diabetes patients requiring GLM therapy have a cardiovascular risk comparable to non-diabetic patients with a prior MI in all 
ages and among both sexes [1]. These findings applied for the prevalent diagnosis and only in patients requiring GLM therapy. As for the diabetes population, the results of our study extend the findings of Schramm et al. in that we document that patients with diabetes already carry a twofold higher risk of cardiovascular death, from the point in time when GLM therapy is initiated. This further supports the proposal that all patients $\geq 30$ years of age with diabetes requiring GLM therapy should be considered for early aggressive multi-factorial intervention in order to reduce the risk of death and cardiovascular events.

Strengths and limitations This study is based on complete and nationwide data obtained from registers that have been previously validated and shown to be reliable $[8,9,26,27]$. The data cover the entire population of Denmark independently of socioeconomic status, age or participation in specific health insurance programmes. Therefore, the risk of selection bias is avoided; moreover, the study notably includes citizens in and out of the labour market. Our study excluded individuals with MI or diabetes before 1 January 1997, which ensured equal disease duration in both groups. The Danish healthcare system partially reimburses drug expenses, and all Danish pharmacies are required to register all dispensed drug prescriptions, ensuring complete registration. However, this study also has several limitations. Thus we were unable to identify patients on diet-only treatment, so our results are only valid for patients receiving glucose-lowering treatment.

Analysis of the Framingham population demonstrated that diabetes duration influences the risk of coronary mortality rates independently of coexisting risk factors [28]. We were unable to asses the duration of diabetes prior to initiation of GLM in our study. However, it is reasonable to assume that patients starting GLM therapy may have had diabetes for approximately 7 to 10 years $[19,29]$. We were also unable to accurately differentiate between type 1 and type 2 diabetes, but prior studies have shown that the risk of cardiovascular morbidity and mortality appears to be the same, regardless of type of diabetes when adjusted for age and sex [1].

Unfortunately, no adjustments could be made for wellknown risk factors such as hypertension, body mass index, physical activity, lipid disorders, dietary factors, microalbuminuria, blood glucose level and smoking, since the registers do not hold information on these clinical characteristics. Although some studies have indicated that the diabetes-related cardiovascular risk depends on a number of risk factors in diabetes mellitus [30], another study has shown that the degree of atherosclerosis is unrelated to the number of risk factors in diabetes [31]. There are important differences in the inclusion criteria of the study that also need to be addressed. It is well established that patients hospitalised for any disease carry an increased risk of death compared with patients diagnosed under out-of-hospital conditions. [32, 33] The incident diabetes population was not diagnosed in connection with an admission to a hospital. Therefore initial risk in that population is not completely comparable with that in the incident MI population of the study.

The diagnostic criteria for MI changed in 1999, with more sensitive diagnostic markers being introduced (i.e. troponins) during the study period. Nevertheless, this has not resulted in dramatic changes in the prognosis for MI patients, and it is unlikely that this has affected our study [34].

The population of Denmark consists mainly of whites, which makes the generalisability of our results to other ethnic groups uncertain.

Conclusions and implications Patients with incident diabetes carry a twofold higher risk of cardiovascular death compared with the general population. This greater risk, moreover, is present as early as the initiation of GLM treatment.

Patients with incident MI have a high short-term risk of all-cause mortality, cardiovascular death and recurrent MI, which rapidly declines towards a steady level. However, they continue to exhibit an approximately two- to threefold higher cardiovascular risk than the general population. Surprisingly, no difference in risk was demonstrated in the two periods. Therefore, our results emphasise the need for vigilance in secondary prophylactic treatment following hospitalisation for MI.

We also found a persistent high cardiovascular risk in patients with diabetes, underscoring the importance of early and aggressive multi-factorial intervention in patients with diabetes.

Acknowledgements The study was supported by a research grant from The Danish Agency of Science, Technology and Innovation-the Danish Research Council (grant no. 271-08-0944).

Duality of interest A. Vaag is employed by Steno Diabetes Center, which is owned by Novo Nordisk A/S. All other authors declare that there is no duality of interest associated with this manuscript.

\section{References}

1. Schramm TK, Gislason GH, Kober L et al (2008) Diabetes patients requiring glucose-lowering therapy and nondiabetics with a prior myocardial infarction carry the same cardiovascular risk: a population study of 3.3 million people. Circulation 117:1945-1954

2. Haffner SM, Lehto S, Ronnemaa T, Pyorala K, Laakso M (1998) Mortality from coronary heart disease in subjects with type 2 diabetes and in nondiabetic subjects with and without prior myocardial infarction. N Engl J Med 339:229-234 
3. Dale AC, Vatten LJ, Nilsen TI, Midthjell K, Wiseth R (2008) Secular decline in mortality from coronary heart disease in adults with diabetes mellitus: cohort study. BMJ 337:a236

4. Booth GL, Kapral MK, Fung K, Tu JV (2006) Relation between age and cardiovascular disease in men and women with diabetes compared with non-diabetic people: a population-based retrospective cohort study. Lancet 368:29-36

5. Preis SR, Hwang SJ, Coady S et al (2009) Trends in all-cause and cardiovascular disease mortality among women and men with and without diabetes mellitus in the Framingham Heart Study, 1950 to 2005. Circulation 119:1728-1735

6. Thomas RJ, Palumbo PJ, Melton LJ 3rd et al (2003) Trends in the mortality burden associated with diabetes mellitus: a populationbased study in Rochester, Minn, 1970-1994. Arch Intern Med $163: 445-451$

7. International Diabetes Federation Clinical Guidelines Task Force. Global guideline for type 2 diabetes. Available from www.idf.org/ webdata/docs/IDF\%20GGT2D.pdf/, accessed 28 January 2009

8. Andersen TF, Madsen M, Jorgensen J, Mellemkjoer L, Olsen JH (1999) The Danish National Hospital Register. A valuable source of data for modern health sciences. Dan Med Bull 46:263-268

9. Madsen M, Davidsen M, Rasmussen S, Abildstrom SZ, Osler M (2003) The validity of the diagnosis of acute myocardial infarction in routine statistics: a comparison of mortality and hospital discharge data with the Danish MONICA registry. J Clin Epidemiol 56:124-130

10. Andersen HR, Nielsen TT, Rasmussen K et al (2003) A comparison of coronary angioplasty with fibrinolytic therapy in acute myocardial infarction. N Engl J Med 349:733-742

11. Gislason GH, Abildstrom SZ, Rasmussen JN et al (2005) Nationwide trends in the prescription of beta-blockers and angiotensin-converting enzyme inhibitors after myocardial infarction in Denmark, 1995-2002. Scand Cardiovasc J 39:42-49

12. Rasmussen JN, Gislason GH, Abildstrom SZ et al (2005) Statin use after acute myocardial infarction: a nationwide study in Denmark. Br J Clin Pharmacol 60:150-158

13. Kontny F (2001) Improving outcomes in acute coronary syndromes - the FRISC II trial. Clin Cardiol 24:I3-I7

14. Fox KA, Mehta SR, Peters R et al (2004) Benefits and risks of the combination of clopidogrel and aspirin in patients undergoing surgical revascularization for non-ST-elevation acute coronary syndrome: the Clopidogrel in Unstable Angina to Prevent Recurrent Ischemic Events (CURE) Trial. Circulation 110:1202-1208

15. Gislason GH, Rasmussen JN, Abildstrom SZ et al (2006) Longterm compliance with beta-blockers, angiotensin-converting enzyme inhibitors, and statins after acute myocardial infarction. Eur Heart J 27:1153-1158

16. Lee HY, Cooke CE, Robertson TA (2008) Use of secondary prevention drug therapy in patients with acute coronary syndrome after hospital discharge. J Manag Care Pharm 14:271-280

17. Malacrida R, Genoni M, Maggioni AP et al (1998) A comparison of the early outcome of acute myocardial infarction in women and men. The Third International Study of Infarct Survival Collaborative Group. New Engl J Med 338:8-14
18. Dominguez H, Schramm TK, Norgaard ML et al (2009) Initiation and persistence to statin treatment in patients with diabetes receiving glucose-lowering medications 1997-2006. Open Cardiovasc Med J 3:152-159

19. Turner RC, Cull CA, Frighi V, Holman RR (1999) Glycemic control with diet, sulfonylurea, metformin, or insulin in patients with type 2 diabetes mellitus: progressive requirement for multiple therapies (UKPDS 49). UK Prospective Diabetes Study (UKPDS) Group. JAMA 281:2005-2012

20. Holman RR, Paul SK, Bethel MA, Matthews DR, Neil HA (2008) 10-year follow-up of intensive glucose control in type 2 diabetes. N Engl J Med 359:1577-1589

21. Gaede P, Vedel P, Larsen N, Jensen GV, Parving HH, Pedersen O (2003) Multifactorial intervention and cardiovascular disease in patients with type 2 diabetes. N Engl J Med 348:383-393

22. Gaede P, Lund-Andersen H, Parving HH, Pedersen O (2008) Effect of a multifactorial intervention on mortality in type 2 diabetes. N Engl J Med 358:580-591

23. Gerstein HC, Miller ME, Byington RP et al (2008) Effects of intensive glucose lowering in type 2 diabetes. $\mathrm{N}$ Engl $\mathrm{J}$ Med 358:2545-2559

24. Duckworth W, Abraira C, Moritz T et al (2009) Glucose control and vascular complications in veterans with type 2 diabetes. N Engl J Med 360:129-139

25. Patel A, MacMahon S, Chalmers J et al (2008) Intensive blood glucose control and vascular outcomes in patients with type 2 diabetes. N Engl J Med 358:2560-2572

26. Krarup LH, Boysen G, Janjua H, Prescott E, Truelsen T (2007) Validity of stroke diagnoses in a National Register of Patients. Neuroepidemiology 28:150-154

27. Gaist D, Sorensen HT, Hallas J (1997) The Danish prescription registries. Dan Med Bull 44:445-448

28. Fox CS, Sullivan L, D'Agostino RB Sr, Wilson PW (2004) The significant effect of diabetes duration on coronary heart disease mortality: the Framingham Heart Study. Diab Care 27:704-708

29. Harris MI, Klein R, Welborn TA, Knuiman MW (1992) Onset of NIDDM occurs at least 4-7 yr before clinical diagnosis. Diab Care 15:815-819

30. Howard BV, Best LG, Galloway JM et al (2006) Coronary heart disease risk equivalence in diabetes depends on concomitant risk factors. Diab Care 29:391-397

31. Scognamiglio R, Negut C, Ramondo A, Tiengo A, Avogaro A (2006) Detection of coronary artery disease in asymptomatic patients with type 2 diabetes mellitus. J Am Coll Cardiol 47:6571

32. Labarere J, Stone RA, Obrosky DS et al (2007) Comparison of outcomes for low-risk outpatients and inpatients with pneumonia: a propensity-adjusted analysis. Chest 131:480-488

33. American Diabetes Association (2008) Economic costs of diabetes in the U.S. in 2007. Diabetes Care 31:596-615

34. Abildstrom SZ, Rasmussen S, Madsen M (2005) Changes in hospitalization rate and mortality after acute myocardial infarction in Denmark after diagnostic criteria and methods changed. Eur Heart J 26:990-995 